\title{
A novel polymorphism near HLA class II region is associated with spontaneous clearance of HCV and response to interferon treatment in Chinese patients
}

\author{
Yin $\mathrm{Xu}^{1,6}$, Peng Huang ${ }^{1,6}$, Ming Yue ${ }^{2}$, Jing $\mathrm{Su}^{1}$, Hongbo Chen ${ }^{3}$, Mingzhu Chen ${ }^{1}$, Jie Wang ${ }^{4}$, Jun $\mathrm{Li}^{2}$, \\ Zhihang Peng ${ }^{1}$, Yun Zhang ${ }^{5}$ and Rongbin $\mathrm{Yu}^{1}$
}

A recent genome-wide association study (GWAS) has identified the single-nucleotide polymorphism (SNP) rs4273729 in a 100-kbp region comprising human leukocyte antigens (HLAs) class II genes as an important predictor of hepatitis C virus (HCV) clearance in European and African populations. This study was to determine whether this polymorphism is also associated with spontaneous HCV clearance as well as response to interferon treatment in Chinese patients. Thus, 686 chronic HCV carriers, 432 individuals with spontaneous viral clearance and 243 patients with pegylated interferon- $\alpha$ and ribavirin (PEG IFN- $\alpha /$ RBV) treatment were genotyped. The rs 4273729 GG genotype was strongly associated with spontaneous HCV clearance as well as better IFN/RBV treatment response compared with the $\mathrm{GC} / \mathrm{CC}$ genotypes in Chinese Han population (additive model: odds ratio $(\mathrm{OR})=0.62,95 \%$ confidence interval $(95 \% \mathrm{Cl})=0.51-0.76 ; \mathrm{OR}=0.58,95 \% \mathrm{Cl}=0.38-0.88$, respectively). Rs4273729, rs12980275, baseline HCV RNA and platelet level were independent predictors for sustained virological response (SVR). The area under the receiver-operating characteristic curve (AUC) was 0.578 when including rs4273729 alone, but the prediction value was improved significantly $(A U C=0.733)$ when further including rs12980275, baseline viral load and baseline platelet level. In conclusion, the genetic variation of rs4273729 is associated with clearance of $\mathrm{HCV}$ in both the natural course and the treatment process in Chinese Han population. Journal of Human Genetics (2016) 61, 301-305; doi:10.1038/jhg.2015.145; published online 3 December 2015

\section{INTRODUCTION}

Hepatitis C virus (HCV) infection is a major global health issue, affecting an estimated $2.4 \%$ of the world population, whereas China alone accounts for almost $20 \%$ of all cases. ${ }^{1,2}$ HCV infection may be self-limited (viral clearance) or persist, but only $15-25 \%$ of the infected individuals would spontaneously clear the virus. ${ }^{3}$ Among the persist patients, the treatment efficiency varies according to the viral genotype. About $50 \%$ of genotype 1 patients and $70-90 \%$ of genotype $2 / 3$ patients can be successfully treated with pegylated interferon- $\alpha$ and ribavirin (PEG IFN- $\alpha / \mathrm{RBV}$ ) combination therapy, then the rest patients with poor treatment response will develop chronic infection and potentially progress to cirrhosis and hepatocellular carcinoma over the next two decades. ${ }^{4,5}$

The distinct consequences of HCV infection likely depend on a complex host-virus interaction. ${ }^{6}$ Studies have demonstrated that host gene polymorphisms influence both the natural course of hepatitis $\mathrm{C}$ and response to therapy ${ }^{7,8}$ The most well-studied single-nucleotide polymorphisms (SNPs) are located near interlenkin-28B (IL28B) gene and the human leukocyte antigen (HLA) region. ${ }^{9-11} \mathrm{~A}$ recent genome-wide association study (GWAS) and other previous studies have showed that the $H L A-D Q B 1^{*} 03: 01$ allele frequencies were associated with the natural outcomes of HCV infection in multiple populations. ${ }^{12-14}$ The GWAS revealed a significant association between the rs4273729 and spontaneous resolution of HCV infection in European and African populations. ${ }^{14}$ Besides, another study showed that the $H L A-D Q B 1^{*} 03: 01$ allele was a potential predictor of response to HCV treatment. ${ }^{15}$ So far, few studies are available about the association between the rs4273729 and the natural outcomes of HCV infection in the Chinese population. We are also interested in whether the rs4273729 can predict response to PEG IFN- $\alpha /$ RBV therapy in Chinese patients infected with HCV genotype 1. IL28B rs12980275, an SNP strongly associated with HCV outcomes in various populations, was studied as the adjustment variable at the same time. This study was done to investigate the relation between rs4273729 and viral clearance in both natural cohort of $\mathrm{HCV}$-infected individuals and treated patients.

\section{MATERIALS AND METHODS}

Ethics statement

Written informed consent for all testing was obtained from the participants in this study, and the study protocol was checked for ethical issues and approved by the institutional review committee of Nanjing Medical University.

\footnotetext{
${ }^{1}$ Department of Epidemiology, School of Public Health, Nanjing Medical University, Nanjing, China; ${ }^{2}$ Department of Infectious Diseases, The First Affiliated Hospital of Nanjing Medical University, Nanjing, China; ${ }^{3}$ Department of Infectious Diseases, The Jurong People's Hospital, Jurong, China; ${ }^{4}$ Department of Basic and Community Nursing, School of Nursing, Nanjing Medical University, Nanjing, China and ${ }^{5}$ Institute of Epidemiology and Microbiology, Huadong Research Institute for Medicine and Biotechnics, Nanjing, China ${ }^{6}$ These authors contributed equally to this work.

Correspondence: Professor R Yu, Department of Epidemiology, School of Public Health, Nanjing Medical University, Nanjing 211166, China.

E-mail: rongbinyu@njmu.edu.cn

Received 7 September 2015; revised 3 November 2015; accepted 4 November 2015; published online 3 December 2015
} 


\section{Study subjects}

Two populations were enrolled in this study. The first population was a natural cohort of HCV-infected individuals. These populations including 1118 Chinese Han individuals were recruited from six hospital hemodialysis centers, Nanjing compulsory detoxification centers and former paid-blood donors in Jiangsu Province from May 2006 to January 2013. All the subjects were not treated. The detailed information of these subjects has been described in our previous studies. ${ }^{16,17}$ Persistent HCV infection cases were defined as anti-HCV and HCV RNA sero-positive for at least three biochemical tests within consecutive 6 months during follow-up; spontaneous viral clearance cases were anti-HCV sero-positive and clearance of HCV RNA in serum, as demonstrated by $\geqslant 2$ instances separated by a minimum of 6 months. The second population was a treatment cohort of HCV patients. A total of 243 chronic hepatitis $\mathrm{C}$ patients with viral genotype 1 were recruited from the Jurong People's Hospital from January 2011 to October 2013. These patients were treatment-naïve and received PEG IFN- $\alpha$ at a dose of $180 \mu \mathrm{g}$ subcutaneously each week plus oral RBV $600-1000 \mathrm{mg}$ daily for 48 weeks according to the standard guidelines. ${ }^{18}$ Successful treatment was ascertained based on sustained virological response (SVR), defined as the absence of HCV RNA 24 weeks after the cessation of therapy.

All participates were $\geqslant 18$ years old and negative for hepatitis $B$ virus co-infection or other types of liver diseases. Each participant was scheduled for an interview with a structured questionnaire to collect information on demographic data and environmental exposure history. After interview, an approximately $5 \mathrm{ml}$ of venous blood sample was collected from each participant. The serum and peripheral blood mononuclear cells were separated and stored at $-70^{\circ} \mathrm{C}$ until assay.

\section{Laboratory testing}

Total DNA from leukocytes was extracted by the phenol-chloroform method according to a standard protocol. ${ }^{19}$ Serum viral load of all treated patients was quantified at baseline, weeks 4, 12, 24, 48 and 24 after cessation of treatment by Cobas Amplicor HCV Monitor Test (v2.0, Roche, Basel, Switzerland). The polymorphisms of rs4273729 (G>C) and IL28B rs12980275 (A>G) were genotyped by the TaqMan allelic discrimination assay on ABI PRISM 7900HT Sequence Detection system (Applied Biosystems, San Diego, CA, USA). Each genotyping assay contained one pair of primers and one pair of probes (sequences provided in Supplementary Table S1). Two blank controls and five repeated samples were assigned into each 384-well format for quality control. The genotyping results were determined by the allelic discrimination mode of the SDS 2.3 software package (Applied Biosystems, Foster City, CA, USA), and a $100 \%$ concordant was achieved.

\section{Statistical analysis}

All statistical analyses were performed with Stata/SE (V.12.0 for Windows; StataCorp LP, College Station, TX, USA). Two-sample $t$ tests, chi-square $\left(\chi^{2}\right)$ test or Fisher's exact tests were used as appropriate to compare demographic characteristics of individuals. Analysis of the allelic frequencies of the investigated polymorphisms showed that both cohorts were in Hardy-Weinberg equilibrium, which was assessed as a quality control measure. Factors associated with HCV spontaneous clearance and SVR were estimated using multivariate logistic regression analysis. Cochran-Armitage trend test was used to analyze the combined effect of two independent SNPs (rs4273729-C and rs12980275-G). A stepwise regression model was then fit comprises all variables and subsequently reduced using forwards elimination to analyze predictive factors for SVR. Each variable in the final models was explained by odds ratio (OR) and 95\% confidence interval (95\% CI). The predictive models of SVR were presented by receiver-operating characteristic curve and area under the curve (AUC) represented the prediction value. In all analyses, $a P$-value of 0.05 or less was considered as statistically significant.

\section{RESULTS}

Baseline feature of the study populations

The baseline demographic and clinical characteristics of the natural cohort (including 686 persistent carriers and 432 resolvers) were summarized in Table 1 . About $65.0 \%$ of the subjects (727/1118) were infected with HCV genotype 1 . The elder, female, blood donors and subjects infected with viral genotype $1 \mathrm{~b}$ were more likely to be chronic carriers $(P<0.05)$. Occurrence of abnormal aspartate aminotransferase (AST) and alanine aminotransferase (ALT) level was higher in the persistent infection group than in the spontaneous clearance group $(P<0.001)$. The rs4273729 minor allele $\mathrm{C}$ was more frequent in the persistent infection group (34.6\%) compared with the clearance group (27.6\%), suggesting that the $\mathrm{C}$ mutation might increase the risk of chronic infection.

The baseline demographic and clinical characteristics of the treatment cohort were shown in Table 2. The mean age of the 243 patients was $53 \pm 8$ years, and $27.8 \%$ of them were males. One hundred fifty-six patients achieved SVR, and the rate was $64.2 \%$. Baseline levels of viral load, albumin, platelets and AFP (alpha-fetal protein) were different between the SVR group and the non-SVR group $(P<0.05)$. Patients with high viral load/AFP and low albumin/ platelet levels at baseline were more likely to fail in treatment. The minor allele rs4273729 C was more frequent in the non-SVR group, further suggesting that the $C$ mutation might be a disadvantage for treatment. Because IL28B rs12980275 has been found to be highly associated with treatment response, ${ }^{10,20}$ it was also analyzed in this study as an adjustment variable. Consistent with the previous studies, the rs12980275 G allele increased the chance of non-SVR (Table 2).

\section{Association of rs4273729 with HCV spontaneous clearance}

Three genetic models (dominant, recessive and additive models) were used to determine the association of rs4273729 with spontaneous viral clearance (Table 3). Spontaneous clearance of HCV was less common in subjects carrying the CC genotype than the GG/GC genotypes (additive model: $\mathrm{OR}=0.62,95 \% \mathrm{CI}=0.51-0.76$ ) after adjustment for age, gender, high-risk population and viral genotype. The results did not show any association between IL28B rs12980275 variants and spontaneous clearance. Differences of age and viral genotype were observed in the rate of spontaneous viral clearance in logistic regression analysis. The risk of susceptibility to HCV chronic infection likely to increase in the subgroups of older $(\geqslant 50$ years, $\mathrm{OR}=0.72$,

Table 1 Characteristics of participants with persistent hepatitis $\mathrm{C}$ virus $(\mathrm{HCV})$ versus those with $\mathrm{HCV}$ clearance

\begin{tabular}{|c|c|c|c|}
\hline Variables & $\begin{array}{c}\text { Persistence } \\
(n=686), N(\%)\end{array}$ & $\begin{array}{c}\text { Clearance } \\
(n=432), N(\%)\end{array}$ & P-value \\
\hline Age $(\geqslant 50$ years $)$ & $416(60.6)$ & $182(42.1)$ & $<0.001$ \\
\hline Male & $271(39.5)$ & $197(45.6)$ & 0.039 \\
\hline High-risk population & & & $<0.001$ \\
\hline HD patients & $84(12.3)$ & $100(23.2)$ & \\
\hline Drug users & $147(21.4)$ & $160(37.0)$ & \\
\hline Blood donors & $455(66.3)$ & $172(39.8)$ & \\
\hline Viral genotype & & & $<0.001$ \\
\hline 1 & $389(56.7)$ & $338(78.2)$ & \\
\hline Non-1 & 297 (43.3) & $94(21.8)$ & \\
\hline AST $\left(\geqslant 40 \cup^{-1}\right)$ & 265 (38.6) & $120(27.8)$ & $<0.001$ \\
\hline $\operatorname{ALT}\left(\geqslant 40 \cup^{-1}\right)$ & $279(40.7)$ & $117(27.1)$ & $<0.001$ \\
\hline \multicolumn{4}{|l|}{ Minor allele (\%) } \\
\hline rs4273729 (C) & $475(34.6)$ & $238(27.6)$ & $<0.001$ \\
\hline rs12980275 (G) & 109 (7.9) & $55(6.4)$ & 0.163 \\
\hline
\end{tabular}

Abbreviations: ALT, alanine aminotransferase; AST, aspartate transaminase; HD patient, hemodialysis patient; Non-1, including genotypes 2, 3 and 2/3.

Rate is calculated in accordance with column. 
$95 \% \mathrm{CI}=0.54-0.95)$ and viral genotype non-1 $(\mathrm{OR}=0.32,95 \%$ $\mathrm{CI}=0.24-0.43)$ (data not shown).

Association of rs4273729 with virological response to treatment For patients with the rs4273729 GG genotype, a higher SVR rate (69.7\%) was observed in comparison with the GC and CC genotypes (62.9 and 41.7\%, respectively) (Table 3). The results also confirmed that subjects with the rs12980275 AA genotype had higher SVR rate than those with homozygous or heterozygous $G$ genotypes (Supplementary Table S2). Then, a stepwise regression model comprises all variables was established, as shown in Table 4, the final model including the rs4273729, rs12980275, baseline HCV RNA level and baseline platelet level as independent predictors of SVR.

Table 2 Baseline characteristics of the patients treated with IFN/RBV

\begin{tabular}{|c|c|c|c|}
\hline Variables & Non-SVR $(\mathrm{n}=87)$ & $\operatorname{SVR}(\mathrm{n}=156)$ & P-value \\
\hline Mean age (years) & $53.29 \pm 8.71$ & $53.02 \pm 7.71$ & 0.808 \\
\hline Male (\%) & $21(24.1)$ & $44(28.2)$ & 0.492 \\
\hline AST $\geqslant 40 \cup I^{-1}(\%)$ & $59(67.8)$ & $94(60.3)$ & 0.242 \\
\hline ALT $\geqslant 40 \mathrm{UI}^{-1}(\%)$ & $55(63.2)$ & $83(53.2)$ & 0.131 \\
\hline Log HCV RNA & $6.26 \pm 0.74$ & $6.00 \pm 0.87$ & 0.023 \\
\hline $\mathrm{TP}\left(\left.\mathrm{g}\right|^{-1}\right)$ & $77.86 \pm 6.04$ & $78.52 \pm 6.16$ & 0.417 \\
\hline Albumin $\left(\left.g\right|^{-1}\right)$ & $42.27 \pm 4.13$ & $43.71 \pm 4.25$ & 0.012 \\
\hline Platelets ( $10^{9}$ per liter) & $118.31 \pm 60.07$ & $136.37 \pm 54.98$ & 0.018 \\
\hline WBC ( $10^{9}$ per liter $)$ & $4.57 \pm 1.69$ & $4.79 \pm 1.51$ & 0.302 \\
\hline Hemoglobin $\left(\left.g\right|^{-1}\right)$ & $130.52 \pm 18.41$ & $134.06 \pm 17.05$ & 0.133 \\
\hline AFP $\left(n g \mathrm{ml}^{-1}\right)$ & $12.15 \pm 19.21$ & $5.01 \pm 7.17$ & 0.001 \\
\hline \multicolumn{4}{|l|}{ Minor allele (\%) } \\
\hline rs4273729 (C) & $64(36.8)$ & $81(26.0)$ & 0.012 \\
\hline rs12980275 (G) & $35(20.1)$ & $27(8.7)$ & $<0.001$ \\
\hline
\end{tabular}

Abbreviations: AFP, alpha-fetal protein; ALT, alanine aminotransferase; AST, aspartate transaminase; HCV, hepatitis C virus; IFN, interferon; Non-SVR, non-sustained virological response; RBV, ribavirin; SVR, sustained virological response; TP, total protein; WBC, white blood cell.

Rate is calculated in accordance with column.
Subsequently, we evaluated combined effects by adding up the number of variant alleles of the two SNPs. The results showed that SVR rate decreased when patients carrying more unfavorable rs4273729 C and rs12980275 G alleles (Figure 1; Supplementary Table S2).

The receiver-operating characteristic analysis for SVR was performed to evaluate the prediction values of the independent factors. When including one SNP of rs4273729, a relatively small area was yielded (AUC $=0.578$ ). Including one SNP of rs12980275 could yield a bigger AUC of 0.641 , suggesting that the prediction value of rs4273729 was weaker than rs12980275. When adding up the four factors of rs4273729, rs12980275, baseline HCV RNA level and baseline platelet level, the prediction value was improved to AUC of 0.733 .

Association of rs4273729 with viral kinetics during therapy The influence of rs4273729 on viral kinetics was further analyzed. Baseline viral load was higher in patients with the GG genotype than in those carrying the $\mathrm{C}$ allele (mean log HCV RNA: 6.17 for GG, 6.03 for GC and 5.98 in CC, $P<0.001)$. However, the drop in viral load was significantly faster in GG patients than in GC/CC patients during the whole treatment (Figure 2). The results might somehow explain

Table 4 Multivariate stepwise regression analysis for independent factors of SVR

\begin{tabular}{lllll}
\hline Variables & Coef. & s.e. & OR $(95 \% \text { Cl })^{a}$ & P-value \\
\hline rs4273729 & -0.49 & 0.21 & $0.61(0.40-0.93)$ & 0.022 \\
rs12980275 & -0.91 & 0.26 & $0.39(0.23-0.67)$ & 0.001 \\
Log HCV RNA & -0.47 & 0.18 & $0.62(0.43-0.89)$ & 0.010 \\
Platelets & -0.01 & 0.003 & $1.01(1.00-1.02)$ & 0.036 \\
\hline
\end{tabular}

Abbreviations: $\mathrm{Cl}$, confidence interval; Coef, coefficient of variation; $\mathrm{HCV}$, hepatitis $\mathrm{C}$ virus; $\mathrm{OR}$, odds ratio; SVR, sustained virological response.

ORs were calculated in additive model.

Table 3 Association of SNP rs4273729 with HCV clearance and SVR

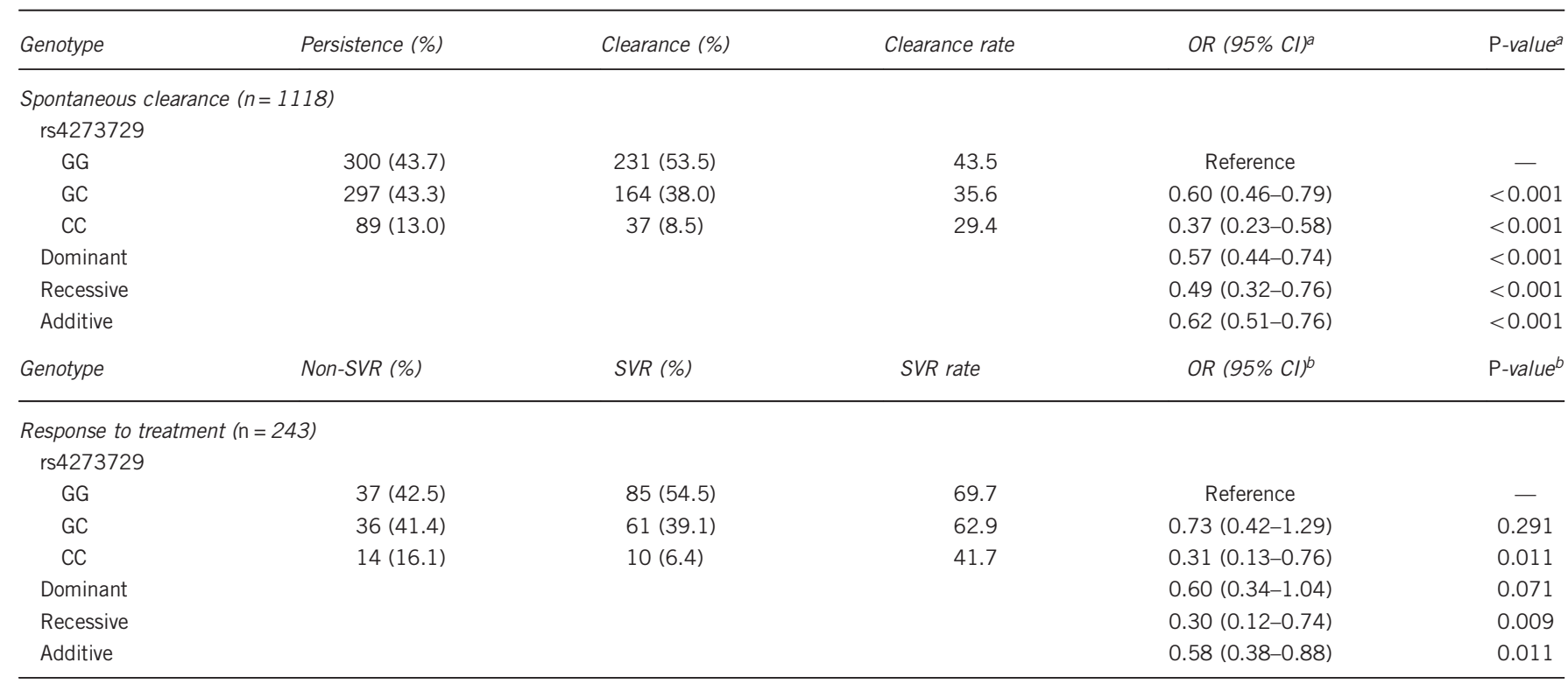

Abbreviations: N-SVR, non-sustained virological response; SVR, sustained virological response.

a Logistic regression analyses adjusted for age, gender, high-risk population and viral genotype.

bLogistic regression analyses adjusted for age, gender, HCV RNA and platelets. 


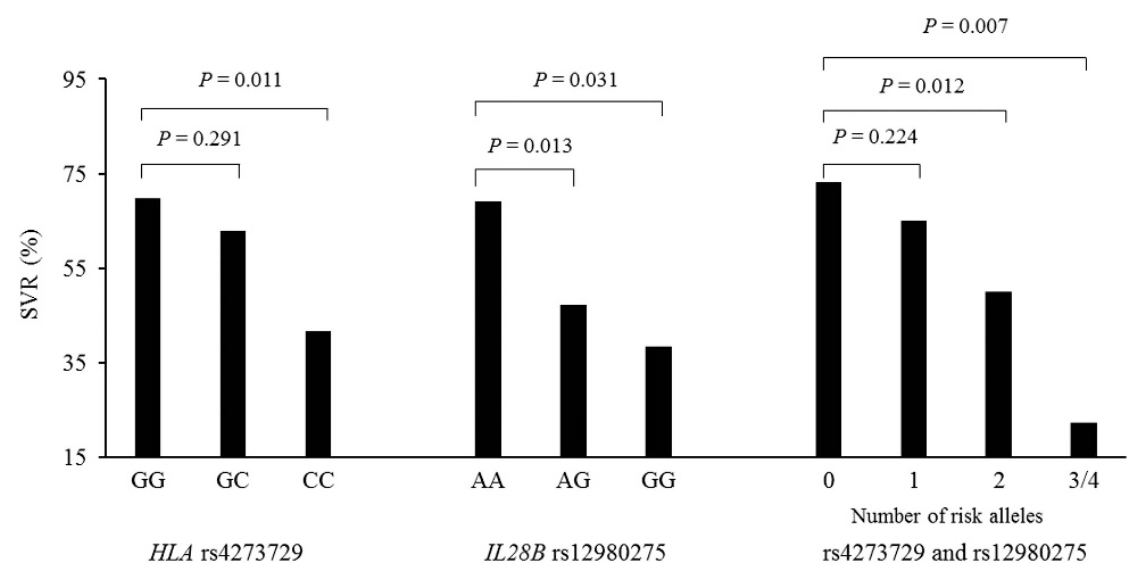

Figure 1 Codominant relationship for SVR for HLA class II and IL28B SNPs. Rate of SVR was compared among patients with different genetic variants. The combined effect of two independent SNPs was analyzed by Cochran-Armitage trend test. Variables are numbers of combined unfavorable genotypes (rs4273729-C and rs12980275-G). Logistic regression analyses were adjusted for age, gender, HCV RNA and platelets.

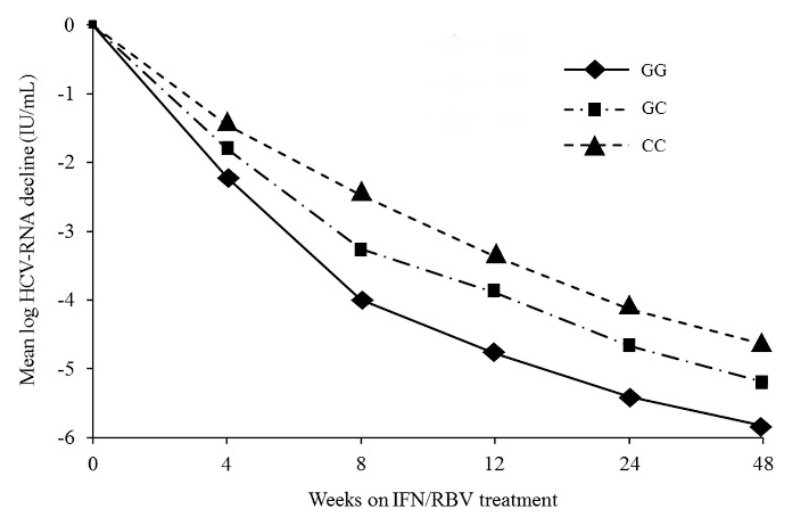

Figure 2 Effect of rs4273729 variants on HCV viral kinetics during therapy. The fold of viral decline was compared among patients with the GG genotype (diamond), the GC genotype (square) and the CC genotype (triangle). The fold of viral decline was calculated as the viral load at followup time point divided by the initial viral load.

why the patients with protective $G$ allele were more likely to achieve SVR.

\section{DISCUSSION}

It has been well established that HLA class II genotypes have an important role in regulation of immune response to HCV infection and are related with spontaneous viral clearance. ${ }^{21,22}$ Our present study is the first to validate the associations of rs4273729 in HLA class II region with the clearance of HCV among high-risk Chinese Han populations with a relatively large sample size. Moreover, we further explored the connection of rs4273729 with the treatment response. It is rare to study the association between HLA rs4273729 and HCV clearance, both spontaneous and INF- $\alpha$ induced in one study. The results of this study confirmed that rs4273729 G allele was associated with increased spontaneous clearance of $\mathrm{HCV}$ in Chinese Han population, which was consistent with the GWAS. ${ }^{14}$ However, our current study found no association of rs12980275 with spontaneous clearance of HCV, but showed the same trend that major allele was associated with spontaneous clearance of HCV, as shown in the previous studies. In this case, one possible explanation is the limited sample size, which was not enough to identify the significant association; and this result may suggested the existence of some confounding factors such as population stratification. In addition, the results showed that the $\mathrm{C}$ mutation was a risk factor of PEG IFN- $\alpha$ / RBV treatment in Chinese patients. The rate of SVR was lower and the viral load decreased more slowly in the patients with $\mathrm{C}$ allele. However, a research of 197 HCV/HIV coinfected patients demonstrated that rs4273729 did not significantly affect the chances of achieving SVR. ${ }^{23}$ The reason might be due to the co-infection that complicated the treatment process. What's more, our treatment cohort had better representativeness because all the patients were paid-blood donors and from the same district, and the sample size was relative larger in this study (243 patients). Therefore, we had confidence in our positive results of rs4273729.

The influence of rs4273729 response to hepatitis C treatment was independent of IL28B rs12980275 (Table 4). The results also confirmed the protective effect of rs12980275 A allele. However, the prediction value of $\mathrm{r} 4273729 \quad(\mathrm{AUC}=0.578)$ was smaller than rs12980275 (AUC $=0.641)$. It seems that so far IL28B is still the most promising factor for prognosis, even though the prediction value of rs12980275 was limited. The results are acceptable because it would be too simple to be true that the treatment outcome is determined by one single genetic allele. Combining more factors would increase the prediction efficiency. In this study, when including four factors of rs4273729, rs12980275, baseline viral load and baseline platelet level, the AUC was improved to 0.733 . The predictive model established in this study was similar to that in previous studies. ${ }^{24,25}$ The results indicated that making full use of routinely collected data could help with prognosis determination and adjustment of therapy procedure.

Several potential limitations need to be taken into consideration in this study. First, we only determined just one SNP in the HLA class II region. However, the $\mathrm{MHC}$ region encompasses complex and extended linkage disequilibrium structure. It suggests the limitation of a single SNP typing as well as the importance of typing HLA themselves. Second, the associations between rs4273729 polymorphism and treatment response of HCV were derived from biologically based hypothesis; therefore, our initial findings need to be further independently verified in different ethnic populations, and further studies should contain other well-known predictive factors such as of treatment response drug adherence and HCV core amino acid 70. Finally, nowadays it is the era of direct antiviral (DAA) but our study only predicted the response to IFN-based therapy. However, in some developing countries, like China, very few DAAs were received regulatory approval because of economic pressures and its unknown 
long-term side effects. According to Chinese actual situation, PEG IFN plus RBV combination therapy is still the standard treatment for hepatitis C. Thus, researches on IFN-based therapy still have certain significance in China. Nevertheless, DAA will be a direction of future research.

In summary, this study showed that the genetic variants of rs4273729 may have an important role in both spontaneous clearance of HCV and viral clearance during treatment in the Chinese population. Studies have demonstrated that patients induced with higher levels of $\mathrm{CD}^{+} \mathrm{T}$ cell were more likely to achieve SVR. ${ }^{26,27}$ Therefore, we assume that the rs4273729 variants might affect the $\mathrm{CD}^{+}{ }^{+} \mathrm{T}$-cell response to $\mathrm{HCV}$ infection. Further studies are needed to elucidate the mechanisms of the SNP.

\section{CONFLICT OF INTEREST}

The authors declare no conflict of interest.

\section{ACKNOWLEDGEMENTS}

The current study was supported in part by National Natural Science Foundation of China (Nos. 81473029, 81502853, 81273146 and 81473028), Research and Innovation Project for College Graduates of Jiangsu Province of China (KYZZ_0265), Natural Science Foundation of Jiangsu Province (BK20151026), Priority Academic Program Development of Jiangsu Higher Education Institutions (PAPD), Medical Reform Project of Health and Family Planning Commission of Jiangsu Province of China (YG201413), Science and Technology Program of Zhenjiang, China (SH20141) and Tianqing Liver Disease Research Supporting Foundation of China Hepatitis Foundation (TQGB20140014). We thank the students who have worked on the study, including Yuangyuan Zhang, Hui Zheng, Wenzhe Ma, Wenjuan Zhang and Xuecheng Song. We also thank the doctors and the nurses from the Jurong Peoples's Hospital for helping with sample collection and organization of the field investigation. This research would not have been possible without the consent and help of the participants.

1 Lavanchy, D. Evolving epidemiology of hepatitis C virus. Clin. Microbiol. Infect. 17, 107-115 (2011).

2 Gower, E., Estes, C., Blach, S., Razavi-Shearer, K. \& Razavi, H. Global epidemiology and genotype distribution of the hepatitis C virus infection. J. Hepatol. 61, S45-S57 (2014).

3 Villano, S. A., Vlahov, D., Nelson, K. E., Cohn, S. \& Thomas, D. L. Persistence of viremia and the importance of long-term follow-up after acute hepatitis $C$ infection. Hepatology 29, 908-914 (1999).

4 Thomas, D. L., Astemborski, J., Rai, R. M., Anania, F. A., Schaeffer, M., Galai, N. et al. The natural history of hepatitis $\mathrm{C}$ virus infection, host, viral, and environmental factors. JAMA 284, 450-456 (2000).

5 Zeuzem, S., Berg, T., Moeller, B., Hinrichsen, H., Mauss, S., Wedemeyer, H. et al. Expert opinion on the treatment of patients with chronic hepatitis C. J. Viral Hepatitis 16, 75-90 (2009).

6 Thimme, R., Bukh, J., Spangenberg, H. C., Wieland, S., Pemberton, J., Steiger, C. et al. Viral and immunological determinants of hepatitis $C$ virus clearance, persistence, and disease. Proc. Natl Acad. Sci. USA 99, 15661-15668 (2002).
7 Thio, C. L. Host genetic factors and antiviral immune responses to hepatitis $C$ virus. Clin. Liver Dis. 12, 713-726 (2008).

8 Yee, L. Host genetic determinants in hepatitis C virus infection. Genes Immun. 5 237-245 (2004).

9 Ge, D., Fellay, J., Thompson, A. J., Simon, J. S., Shianna, K. V., Urban, T. J. et al. Genetic variation in IL28B predicts hepatitis C treatment-induced viral clearance. Nature 461, 399-401 (2009).

10 Tanaka, Y., Nishida, N., Sugiyama, M., Kurosaki, M., Matsuura, K., Sakamoto, N. et al. Genome-wide association of IL28B with response to pegylated interferon- $\alpha$ and ribavirin therapy for chronic hepatitis C. Nat. Genet. 41, 1105-1109 (2009).

11 Tamori, A. \& Kawada, N. HLA class II associated with outcomes of hepatitis B and C infections. World J. Gastroenterol. 19, 5395 (2013).

12 Miki, D., Ochi, H., Takahashi, A., Hayes, C. N., Urabe, Y., Abe, H. et al. HLA-DQB1* 03 confers susceptibility to chronic hepatitis $C$ in Japanese: a genome-wide association study. PLOS ONE 8, e84226 (2013).

13 Yu, R. B., Hong, X., Ding, W. L., Tan, Y. F., Zhang, Y. X., Sun, N. X. et al. The association between the genetic polymorphism of HLA-DQA1, DQB1, and DRB1 and serum alanine aminotransferase levels in chronic hepatitis $\mathrm{C}$ in the Chinese population. J. Gastroenterol. Hepatol. 23, 1394-1402 (2008).

14 Duggal, P., Thio, C. L., Wojcik, G. L., Goedert, J. J., Mangia, A., Latanich, R. et al. Genome-wide association study of spontaneous resolution of hepatitis $C$ virus infection, data from multiple cohorts. Ann. Intern. Med. 158, 235-245 (2013).

15 de Rueda, P. M., López-Nevot, M. Á., Sáenz-López, P., Casado, J., Martín-Casares, A. Palomares, P. et al. Importance of host genetic factors HLA and IL28B as predictors of response to pegylated interferon and ribavirin. Am. J. Gastroenterol. 106, 1246-1254 (2011).

16 Huang, P., Dong, L., Lu, X., Zhang, Y., Chen, H., Wang, J. et al. Genetic variants in antigen presentation-related genes influence susceptibility to hepatitis $C$ virus and viral clearance, a case control study. BMC Infect. Dis. 14, 3837 (2014).

17 Huang, P., Zhang, Y., Lu, X., Xu, Y., Wang, J., Zhang, Y. et al. Association of polymorphisms in HLA antigen presentation-related genes with the outcomes of HCV infection. PLOS ONE 10, e0123513 (2015).

18 Chinese Society of Hepatology, Chinese Society of Infectious Disease and Parasitic Disease. Prevention guide of hepatitis C. Chin. J. Hepatol. 12, 194-198 (2004) [in Chinese].

19 Saldanha, J., Gerlich, W., Lelie, N., Dawson, P., Heermann, K. \& Heath, A. An international collaborative study to establish a World Health Organization international standard for hepatitis B virus DNA nucleic acid amplification techniques. Vox Sanguinis 80, 63-71 (2001).

20 Sarrazin, C., Susser, S., Doehring, A., Lange, C. M., Müller, T., Schlecker, C. et al. Importance of IL28B gene polymorphisms in hepatitis C virus genotype 2 and 3 infected patients. J. Hepatol. 54, 415-421 (2011).

21 Alric, L., Fort, M., Izopet, J., Vinel, J., Charlet, J., Selves, J. et al. Genes of the major histocompatibility complex class II influence the outcome of hepatitis $C$ virus infection. Gastroenterology 113, 1675-1681 (1997).

22 Cramp, M. E., Carucci, P., Underhill, J., Naoumov, N. V., Williams, R. \& Donaldson, P. T. Association between HLA class II genotype and spontaneous clearance of hepatitis C viraemia. J. Hepatol. 29, 207-213 (1998).

23 Franco, S., Tural, C., Clotet, B. \& Martinez, M. A. Alleles at rs4273729 in the chromosome 6 do not predict response to peg-interferon- $\alpha$ and ribavirin therapy in hepatitis C virus/HIV-1 - coinfected patients. AIDS 26, 1973-1974 (2012).

24 Zhang, Y. Y., Chen, H. B., Xu, Y., Huang, P., Wang, J., Zhang, Y. et al. Interferon- $\lambda$ related genes and therapeutic response in Chinese hepatitis $\mathrm{C}$ patients. World $\mathrm{J}$. Gastroenterol. 21, 4006-4013 (2015).

25 Ochi, H., Hayes, C. N., Abe, H., Hayashida, Y., Uchiyama, T., Kamatani, N. et al. Toward the establishment of a prediction system for the personalized treatment of chronic hepatitis C. J. Infect. Dis. 205, 204-210 (2012).

26 Rosen, H. R., Weston, S. J., Im, K., Yang, H., Burton, J. R., Erlich, H. et al. Selective decrease in hepatitis $\mathrm{C}$ virus-specific immunity among African Americans and outcome of antiviral therapy. Hepatology 46, 350-358 (2007).

27 Golden-Mason, L., Klarquist, J., Wahed, A. \& Rosen, H. PD-1 expressin is increased on immunocytes in chronic HCV and predicts failure of response to antiviral therapy, race-dependent differences. J. Immunol. 180, 3637-3641 (2008).

Supplementary Information accompanies the paper on Journal of Human Genetics website (http://www.nature.com/jhg) 